\title{
RELACIÓN DE LA SATISFACCIÓN LABORAL CON ESTILOS DE LIDERAZGO EN ENFERMEROS DE HOSPITALES PÚBLICOS, SANTIAGO, CHILE
}

\author{
RELATION OF THE JOB SATISFACTION WITH LEADERSHIP STYLES IN \\ NURSES OF PUBLIC HOSPITALS, SANTIAGO, CHILE
}

\author{
LisSETTE GonZÁlez Álvarez * \\ Eduardo Guevara Sotomayor ${ }^{* *}$ \\ Guillermo Morales Figueroa *** \\ Paulina Segura Hernández ${ }^{* * * *}$ \\ Carolina Luengo Martínez ${ }^{* * * *}$
}

\begin{abstract}
RESUMEN
La satisfacción laboral es el clásico indicador al que se recurre cuando se desea conocer la actitud general de las personas hacia su vida laboral. Su carencia contribuye al aumento de factores adversos que implican consecuencias costosas para la empresa. Al ejercer liderazgo, las personas adoptan "estilos", que probablemente puedan tener cierta influencia en la Satisfacción Laboral de los trabajadores, lo que puede incidir en el trabajo que desempeñan. Objetivo: Determinar si la Satisfacción Laboral percibida por los profesionales de Enfermería de los Centros de Responsabilidad (CR) o Servicios de 5 Hospitales del sector "Gran Santiago", de la RM, está relacionada a los Estilos de Liderazgo del Modelo de Hersey y Blanchard. Metodología: Estudio cuantitativo, transversal de tipo descriptivo-correlacional. Muestra: 214 enfermeros universitarios. Resultados: Se determinó que la mayoría de los profesionales de Enfermería pertenecientes a los CR o Servicios de medicina y cirugía, se encuentran satisfechos laboralmente, los pertenecientes al CR o Servicio de urgencia, se encuentran insatisfechos; se destaca que el 27,6\% de los enfermeros que declararon sentirse satisfechos laboralmente se relacionan con el estilo entrenador de su superior jerárquico. Conclusión: A pesar que no existe un estilo de liderazgo mejor que otro, sino uno más adecuado para la situación, hay una relación entre la satisfacción laboral con los estilos de liderazgo, motivo por el cual es importante profundizar en estos temas para obtener conocimientos que permitan formar profesionales líderes, capaces de mantener satisfecho a su equipo de trabajo.
\end{abstract}

Palabras clave: Satisfacción laboral, liderazgo, enfermeros, servicios hospitalarios.

\begin{abstract}
The Job Satisfaction is the classic indicator to which it is appealed when one wants to know the general attitude of the persons towards his working life. His lack contributes to the increase of adverse factors that imply costly consequences for the company. On having exercised leadership, the persons adopt "styles", which probably could have certain influence in Job Satisfaction of the workers that can affect the work they are performing. Objective:

\footnotetext{
* Enfermera, Hospital Base Puerto Montt, Chile. E-mail: lis-gonzalez@hotmail.com

** Enfermero, Hospital Herminda Martín de Chillán. E-mail: cono_tab@hotmail.com

${ }^{* * *}$ Enfermero, Hospital Padre Hurtado, Santiago, Chile. E-mail: memorw22@hotmail.com

${ }^{* * * *}$ Enfermera, DESAMU el Carmen, Chile. E-mail: paulii.segura@gmail.com

${ }^{* * * * *}$ Enfermera, docente Departamento de Enfermería, Universidad del Bío Bío Campus Chillán-Chile. E-mail: cluengo@ ubiobio.cl, caroluengo@gmail.com
} 
To determine if the Job Satisfaction perceived by the Centers of Responsibility (CR) Nursing professionals or Services of 5 Hospitals of the sector "Great Santiago" of the Metropolitan Area, is related to the Leadership styles of the model of Hersey and Blanchard. Methodology: descriptive, cross-sectional and correlational study. Sample: 214 university nurses. Results: most nursing professionals belonging to the CR or medical and surgical services, are satisfied upon their work, those in the CR or emergency service, were dissatisfied; It should be noted that $27.6 \%$ of nurses reported being satisfied occupationally related to the coaching leadership style of his hierarchical superior. Conclusion: Although there is no leadership style better than another, but one most appropriate for the situation, there is a relationship between job satisfaction with leadership styles, which is why it is important to look into these topics for knowledge to be leading professionals, able to maintain satisfied the working team.

Key words: Job satisfaction, leadership, nurses, hospitable services.

Fecha recepción: 06/01/12 Fecha aceptación: 15/01/13

\section{INTRODUCCIÓN}

El grado de satisfacción puede afectar la cantidad y calidad del trabajo que desempeñan los sujetos, e incidir en la baja calidad de la prestación, ausentismo laboral, propensión a abandonar la organización, entre otros (1-3). La satisfacción laboral es un aspecto que ha sido ampliamente estudiado desde que Hoppock, ya en el año 1935, publicó la primera investigación que hacía un análisis profundo de la satisfacción laboral, determinando que existen múltiples factores que podrían ejercer influencia sobre la satisfacción laboral como: fatiga, monotonía, condiciones de trabajo y supervisión $(4,5)$. La satisfacción laboral se define como el "grado de placer que el empleado obtiene de su trabajo" (6). En la atención sanitaria ha aumentado en importancia, e incluso se podría decir que al determinar si los profesionales se encuentran o no satisfechos, se está midiendo la calidad de la organización y de sus servicios internos (1, 2). A partir de lo anterior, la satisfacción laboral se entiende como un factor determinante de la calidad de la atención, y un estado emocional positivo o placentero de la percepción subjetiva de las experiencias laborales del sujeto, en el que inciden las circunstancias y características de la labor que se desempeña, y las individualidades de cada trabajador, condicionando la respuesta afectiva de éste hacia diferentes aspectos del trabajo $(3,7)$. Para un individuo, trabajar significa tener un propósito, expresarse y sentirse satisfecho de contribuir a la sociedad $(8,9)$. A raíz de la estrecha relación que existe entre la satisfacción laboral y la productividad, existe un interés creciente en los administradores por mejorar la calidad de vida en el trabajo, especialmente en los profesionales de Enfermería, pues ellos deben velar por la salud física y mental de quienes están a su cargo, y entregar una atención de buena calidad $(1,2)$. Las intervenciones destinadas a mejorar la práctica clínica y salud psicológica de enfermeras y personal del hospital debe centrarse en la carga de trabajo, expectativas personales de los trabajadores y satisfacción en el trabajo (10). Una de las definiciones más usuales y generales de satisfacción en el trabajo, que incluye componentes cognoscitivos y afectivos es la siguiente: "Un estado emocional agradable o positivo que es el resultado de la valoración del trabajo personal o la experiencia que brinda el trabajo" (11). Tiene lugar cuando, en virtud de estar involucrado en ciertas actividades dentro de un ambiente de trabajo particular, las necesidades específicas de un individuo son satisfechas $(7,12)$. En diciembre del año 1997, se incorpora en Chile la profesión enfermera dentro del Código Sanitario, el cual en su ar- 
tículo $\mathrm{N}^{\circ} 113$ establece que "los servicios profesionales de la enfermera/o comprenden la Gestión del Cuidado en lo relativo a promoción, mantención y restauración de la salud, la prevención de enfermedades o lesiones, $y$ la ejecución de acciones derivadas del diagnóstico y tratamiento médico y el deber de velar por la mejor administración de los recursos de asistencia para el paciente (13). En Chile, por tanto, Gestión de los Cuidados significa autonomía, es decir "libertad para actuar en lo que se sabe, considerando el juicio independiente para lograr un resultado deseado". Para llevar a cabo dicha autonomía se requiere de profesionales capaces de practicar liderazgo efectivo, lo cual implica la toma de decisiones, la distribución de los recursos, manejo de conflictos, comunicación y la selección de estrategias adecuadas a fin de motivar a las personas que conforman el equipo multidisciplinar $(1,2)$. En este contexto, el Colegio Internacional de Enfermeras señala: "Las enfermeras han de seleccionar programas adecuados, unidisciplinarios o multidisciplinarios que las preparen eficazmente para la gestión, la elaboración de políticas y el liderazgo en diversos contextos, en las distintas fases de su desarrollo profesional y en la evolución de su carrera" (14). Liderazgo se puede definir como "el proceso de influencia que un individuo ejerce sobre otro individuo o grupo, para lograr el alcance de objetivos en una determinada situación" (1, $2,14)$. En 1948, una investigación llevada a cabo por Stogdill, ampliada y revisada 25 años más tarde, demuestra que no existe un grupo de características que definan universalmente el liderazgo (15). En 1974 el autor concluyó que las características y habilidades que requiere el liderazgo vienen determinadas por una situación específica (16). Conciente o inconscientemente, el liderazgo se lleva a la práctica de maneras diferentes, dependiendo de la situación, de la disposición de los poderdantes y según las características propias del mismo líder, adoptando "Estilos de Liderazgo" (17). El ejercicio del liderazgo, a pesar de ser complejo, es inherente a la organización del trabajo y repercute en la articulación de los equipos (18). Al efectuar su función, un líder debe acercarse a un estilo en particular y entre éstos destacan, según el modelo de Blanchard y Hersey, el director, entrenador, participativo/soportativo y delegador $(4,13,17)$. Con esto se debe entender que el modelo o estilo adoptado define la eficacia del líder en función de su capacidad para utilizar cada uno de los estilos de acuerdo con la situación de los colaboradores (3, 13). No existe un estilo de liderazgo que sea siempre correcto de utilizar en todas las situaciones (17), por tanto, el líder más eficaz será aquel que demuestre una mayor flexibilidad de estilo y que utilice el estilo adecuado con la persona adecuada. Según Hersey et al. (19), a cada nivel de preparación le correspondería un estilo de liderazgo en concreto. El modelo de Hersey y Blanchard ha contribuido a que profesionales y consultores de todo el mundo prestaran una mayor atención al concepto "situación". El Modelo de Liderazgo Situacional comprende y explica la interacción existente entre las dimensiones de comportamiento de tarea y de relación junto con el nivel de madurez o preparación de los seguidores a la hora de desempeñar un rol u objetivo, es decir, incluye tres componentes básicos, dentro de los que se encuentra una "descripción de las situaciones alternativas que podrían encontrar los líderes, una serie de posibles estilos de liderazgo y recomendaciones sobre qué estilo de liderazgo es más apropiado adoptar en determinadas circunstancias" (19-21). Es indispensable para el ejercicio del liderazgo contar con seguidores de personas sobre las cuales ejercer influencia para conseguir objetivos comunes (3). En este sentido podemos decir que la esencia del liderazgo son los seguidores, lo que hace que una persona sea líder es la disposición de la gente a seguirla (22) .Y a nivel empresarial es de vital importancia que éstos gocen, entre otras cosas, de buena satisfacción laboral, ya que su carencia 
contribuye al aumento de factores adversos, que implican consecuencias costosas para la empresa (3), los cuales se podrían ver reducidos si los líderes de Enfermería favorecen la satisfacción en el trabajo a través de una adecuada organización y gestión $(1,2)$. Por tanto, influirá en la Satisfacción Laboral el estilo de liderazgo percibido por los profesionales de Enfermería. Probablemente, en la medida en que cada estilo sea ejercido o no, acorde a la situación, el nivel de satisfacción laboral se verá afectado, premisa bajo la cual se instala el propósito central de esta investigación. De esta forma, al relacionar el estilo utilizado por el líder de Enfermería y la satisfacción de los seguidores respecto a su trabajo, probablemente se pondrán de manifiesto una serie de antecedentes y/o estrategias que permitan la creación de un buen clima laboral, en el cual prime la comunicación y coordinación entre puestos jerárquicos y enfermeros, el cumplimento de objetivos, metas y resultados esperados al tener equipos de salud satisfechos, la identificación de metodologías y planes que fomenten la confianza del grupo, el desarrollo de las capacidades del personal de trabajo, la valoración del profesional de Enfermería como miembro importante del equipo multidisciplinar, así como también su participación activa en todas las áreas de desempeño profesional. Mantener niveles altos de satisfacción laboral permite mejorar los procesos, fomenta el trabajo en equipo, aumenta la calidad de la atención de Enfermería y el rendimiento de su productividad, así como la satisfacción de los usuarios (23).

\section{Planteamiento del problema}

La elección de un estilo de liderazgo en particular podría ser un factor de gran relevancia en el aumento de la Satisfacción Laboral de los seguidores, de manera que cada uno de ellos pueda lograr el máximo desarollo posible de sus habilidades intrínsecas, alcanzando así una alta eficiencia para ellos mismos y para su equipo. Tanto a nivel local como nacional existe un bajo nivel de información con respecto a Satisfacción Laboral de Enfermería y su relación con los estilos de liderazgo, como lo ratifica un estudio realizado en Etiopía, donde se concluye que el estilo de liderazgo de enfermeras gerentes juega un papel importante en la satisfacción laboral de enfermeras. Sin embargo, hay literatura limitada en áreas relacionadas con el estilo de liderazgo de gerente de personal de Enfermería (24). Motivo que brinda una excelente oportunidad para generar conocimientos científicos actuales, que se traduzcan en un incentivo para el sector educativo de formar profesionales de Enfermería líderes, capaces de enfrentar de manera óptima los nuevos desafíos que impone el medio, ratificando nuestra autonomía como profesionales.

\section{Objetivo general}

Determinar si la Satisfacción Laboral percibida por los profesionales de Enfermería de los Centros de Responsabilidad (CR) o Servicios de 5 Hospitales del sector "Gran Santiago" de la Región Metropolitana está relacionada a los Estilos de Liderazgo.

\section{Variables}

Variable dependiente: Satisfacción laboral.

Variable independiente: Estilos de liderazgo.

Variables de estratificación: Centros de Responsabilidad o Servicios Clínicos, tipo de usuario que atienden.

\section{MATERIAL Y MÉTODO}

Estudio de carácter cuantitativo, descriptivocorrelacional de corte transversal. Se estudiaron a profesionales de Enfermería con título universitario de Enfermero(a) con cargo de 
"Enfermera/o Clínica/o", de los servicios de Medicina, Cirugía y Urgencia pertenecientes a cinco hospitales de alta complejidad ubicados en la Región Metropolitana (RM) de Santiago de Chile. Estos centros hospitalarios son considerados hospitales tipo 1, establecimientos ubicados en ciudades con más de 500.000 habitantes, cuentan con alrededor de 500 camas, además tienen adosado un centro de diagnóstico terapéutico, un servicio de urgencia organizado, residencia interna diferenciada por servicio clínico y unidades de tratamiento intensivo (25).

Se diseñó un muestreo por conglomerado, seleccionándose aleatoriamente a cinco hospitales (unidades de muestreo) de los 17 existentes en la RM, de los cuales se estudió a la totalidad de enfermeros (unidad de análisis) en cada unidad de muestreo. Se excluyeron a enfermeros que se encontraban con licencia médica, vacaciones, día administrativo por no estar en el servicio durante el período de recolección de datos; se excluyeron además a quienes negaron dar su consentimiento, aquellos que se encontraban realizando reemplazos o que tenían menos de 3 meses trabajando en dichos servicios hospitalarios, por lo cual se totalizó una muestra de 214 individuos. Los datos se obtuvieron a través de la autoaplicación de un cuestionario que incluye dos instrumentos, uno de ellos, que permite valorar la Satisfacción Laboral, extraído del Instituto Nacional de Seguridad e Higiene del Trabajo de España (26, 27). Se basa en los cuestionarios elaborados primeramente por el Survey Research Center, de Michigan; en los trabajos posteriores de Herzberg y Crozier en los EE.UU. y Francia, respectivamente (1966-1970) y en adaptaciones españolas $(26,27)$. Al igual que en el estudio desarrollado en Argentina denominado "Satisfacción laboral en el personal de enfermería del sector público" (27) se utilizó sólo la primera parte para describir un índice cuantitativo general y específico de satisfacción laboral -SL- breve, fácilmente puntuable y con posibilidad de aplicación colecti- va, cuyas escalas utilizadas fueron validadas por diversos autores en estudios realizados en distintos países (26). Consta de siete preguntas adaptadas por el mismo Instituto al idioma español. La valoración del cuestionario se realizó en cuatro formas diferentes en relación con el grado de insatisfacción, M, B, P y N (muy satisfecho, 4 pts; bastante satisfecho, 3 pts; poco satisfecho, 2 pts; nada satisfecho, 1 pto.). Para efectos de este estudio, una vez aplicado el cuestionario y realizando el análisis de resultados, se decidió reducir de las cuatro posibilidades originales de Satisfacción Laboral a dos nuevas categorías, debido a que no fue posible determinar una prueba de independencia por la presencia de celdas con valor cero y frecuencias esperadas menores a cinco. Es así como los iniciales niveles poco y nada satisfechos se categorizaron como "insatisfechos", mientras que los bastante y muy satisfechos se agruparon para dar origen a la categoría "satisfechos". Y el otro utilizado con el fin de medir los distintos estilos de liderazgo, llamado SBDQ (Supervisory Behavior Description Questionnaire) (21), el cual presenta índices de fiabilidad y validez adecuados con un alfa de Cronbach de 0,92 obtenido en un estudio mexicano (28). Este instrumento procede de los estudios sobre el liderazgo de la Universidad de Ohio y en las últimas décadas ha sido empleado en investigación en diferentes organizaciones de forma extensa. Consta de 48 ítemes, cada uno de ellos con 5 alternativas de respuesta, que describen la conducta del superior. Los primeros 28 ítemes miden la dimensión relación. Los 20 ítemes restantes miden la dimensión tarea. Se solicitó mediante carta adjunta el consentimiento de dichos profesionales, conservando anonimato y confidencialidad, respetando la libertad del individuo en cualquier momento del estudio. Previo a lo anterior se solicitó autorización para la aplicación de los instrumentos de investigación a las/los directoras/es de los hospitales en estudio y a los respectivos Comités de Ética de cada establecimiento que lo 
requirieron o a la enfermera coordinadora, según el caso de cada establecimiento.

Análisis estadístico: se describieron las variables del estudio mediante el cálculo de las frecuencias observadas y porcentuales que contengan la característica de interés para aquellas variables de tipo nominal $u$ ordinal. Para asociar variables de tipo nominales $\mathrm{u}$ ordinales se recurrió a la prueba de independencia $\mathrm{Chi}^{2}$. Para graficar las variables en estudio se recurrió a la técnica estadística de Análisis por Correspondencia con normalización simétrica, dispuesta en ejes cartesianos de dos dimensiones. En todos los análisis estadísticos se asume un nivel de significación de 0,05. Para todo el trabajo estadístico se trabajó en el programa SPSS versión 14.0.

\section{RESULTADOS}

De los 214 enfermeros de ambos sexos estudiados, el $65 \%$ atendieron en hospitales que entregan servicios a adultos $(n=139)$, mientras que el restante $35 \%$ atienden en hospitales especializados en niños $(n=75)$. Se destaca que el 2,3\% de la muestra no se encuentra satisfecho en su trabajo $(n=5)$, mientras que el $39,8 \%$ es catalogado como poco satisfecho $(n=84)$, por otro lado, un $55,6 \%$ está bastante satisfecho $(n=119)$ y un porcentaje no mayor al 3\% es agrupado dentro de la categoría de muy satisfecho $(2,8 \%)$. Respecto a la distribución de la muestra según servicios hospitalarios donde laboran los profesionales de Enfermería, casi la mitad de los sujetos estudiados trabajan en los servicios de Medicina $(49,1 \% ; n=105)$, mientras que el $30,4 \%$ $(n=65)$ y el $20,6 \%(n=44)$ de las y los enfermeros trabajan en los servicios de Cirugía y Urgencia, respectivamente.

Respecto de los estilos de liderazgo de los profesionales de Enfermería, se observa que la mayoría de los profesionales de Enfermería perciben el estilo de liderazgo "Entrena- dor" (37,9\%), seguido por el estilo "Delegador" (23,4\%), "Participativo" (20,6\%) y en menor cuantía se percibe el estilo "Director".

Al estudiar la correlación entre el estilo de liderazgo y la satisfacción laboral de los enfermeros estudiados, se evidencia que aquellos funcionarios con niveles de satisfacción positivo (muy y bastante satisfecho) son los que laboran en servicios clínicos ligados a cirugía y medicina, mientras que los niveles de baja satisfacción están relacionados con personal de Enfermería que trabaja en Urgencias. Aquellas personas que fueron caratuladas como Nada Satisfecho no se encuentra ligado con ningún servicio clínico en particular, por lo que puede entenderse como un criterio que se observa indistintamente de los tres tipos de servicios clínicos estudiados.

Debido a que no se pudo determinar una prueba de independencia por la presencia de celdas con valor cero y frecuencias esperadas menores a cinco en lo que respecta a la satisfacción laboral, se decidió disminuir de las cuatro posibilidades originales de Satisfacción Laboral a dos nuevas categorías; "Satisfecho" e "Insatisfecho", resultando entonces que el 58,3\% de los profesionales de enfermería se catalogaron como satisfecho laboralmente $(n=125)$, a diferencia de un $41,6 \%$ de quienes están insatisfechos $(n=89)$. Al observar la satisfacción laboral respecto del servicio clínico donde trabajan los enfermeros del estudio, el 52,4\% de la muestra que trabaja en los servicios de Medicina y Cirugía están satisfechos, mientras que el 14,5\% de quienes laboran en el Servicio de Urgencias está insatisfecho, a diferencia de un 6,1\% que se encuentra satisfecho $(n=13)$. Según la prueba de independencia $\mathrm{Chi}^{2}=20,787 ; 2 \mathrm{gl}$; $p<0,001$, existe suficiente evidencia que permite afirmar una asociación significativa entre la satisfacción laboral y el servicio clínico donde labora el profesional de Enfermería, de tal forma que los satisfechos son quienes laboran en servicios clínicos de Medicina y Cirugía, mientras que los insatisfechos se li- 
gan predominantemente con el Servicio de Urgencia.

Al estudiar el tipo de liderazgo percibido por los profesionales de Enfermería estudiados respecto de la satisfacción laboral, los estilos "Delegador" y "Director" se correlacionan con poca y nula satisfacción laboral, respectivamente, mientras que los estilos "Participativo" y "Persuasivo" se relacionan preferentemente con el nivel "bastante satisfecho". Al estudiar la misma variable pero agrupándose las categorías en "insatisfecho" y "satisfecho", el nivel de satisfacción se asocia significativamente con el estilo de liderazgo $\mathrm{Chi}^{2}=26,293 ; 3$ gl; p<0,001, observándose que aquellos profesionales de enfermería insatisfechos laboralmente perciben como estilos de liderazgo predominante: "director" y "delegador", mientras que los sujetos que se encasillaron dentro de la categoría satisfacción laboral perciben un estilo de liderazgo predominante: "persuasivo" y "participativo".

Al incorporar al binomio Satisfacción Laboral y Estilo de liderazgo la variable tipo de usuario, sólo en el estrato de atención de adultos se evidencia una asociación significativa entre las dos variables $\mathrm{Chi}^{2}=23,606$ (3gl) $\mathrm{p}<0,001$. Se observa, además, que el mayor porcentaje de profesionales de Enfermería que brindan atención a usuarios adultos categorizados como satisfechos con un $55,4 \%$ $(\mathrm{n}=77)$ se relaciona preferentemente con un Estilo de liderazgo "Entrenador" (28,1\%), mientras que aquellos categorizados como insatisfechos en el mismo estrato de atención correspondiente a un 44,6\% $(n=62)$, se relacionan con un Estilo de liderazgo "Director" $(17,3 \%)$.

\section{DISCUSIÓN Y CONCLUSIÓN}

Este estudio arrojó que la mayoría de los profesionales de Enfermería que se encuentran bastante y muy satisfechos pertenecen a los servicios de Medicina y Cirugía. Al contra- rio, en el Servicio de Urgencia predomina el nivel de satisfacción poco y nada satisfecho. La percepción de la Satisfacción Laboral (clasificada en satisfecho e insatisfecho), de los profesionales de Enfermería de los servicios de Medicina y Cirugía, es de satisfechos. Por otro lado, en el Servicio de Urgencia los profesionales de Enfermería manifiestan insatisfacción en su trabajo. En contraste a lo evidenciado en esta investigación, RiberaDomene et al. (29) afirman en su estudio que los profesionales de Enfermería sufren una gran frustración profesional, con altas puntuaciones en estados depresivos y corrobora que las enfermeras que brindan sus cuidados en servicios de hospitalización presentan índices mayores de depresión, estrés y frustración profesional y por ende menores niveles de Satisfacción Laboral que aquellas que ejercen su profesión en servicios de Urgencia. Esta contraposición de resultados, sugiere la presencia de nuevos factores que podrían influir en que los profesionales que trabajan en los servicios de Urgencia presenten niveles de Satisfacción Laboral más bajos que aquellos que trabajan en Medicina y Cirugía, como por ejemplo los analizados en el estudio de Ríos y Godoy (30), en donde el reconocimiento profesional, la tensión laboral por la responsabilidad percibida y las posibilidades de promoción profesional se configuran como las áreas deficitarias con la que los profesionales se encuentran menos o insatisfechos. La insatisfacción laboral en los recursos humanos de Enfermería es una condición peligrosa, ya que atender a seres humanos y hacerlo en forma descontenta puede acarrear graves consecuencias para la salud de las personas que necesitan cuidados de salud y, por lo tanto, ser atendidos inadecuadamente (31). Por otro lado, como lo determina es su estudio Díaz et al. (32), la falta de satisfacción laboral sumada a algunas variables individuales actuarían como condicionantes para la aparición del Síndrome de Desgaste Laboral o "Burnout" (BO), enfermedad laboral que afecta principalmente a 
profesionales que brindan servicios.

Respecto a la variable Estilos de liderazgo la mayoría de los profesionales de Enfermería percibe el estilo "Entrenador" y la minoría el estilo "Director". En Chile no existen estudios que profundicen en este tema, por lo que esta evidencia se constituye en un aporte concreto a la disciplina, en su marco de Gestión del Cuidado; como lo indican Véliz y Paravic (22), en las organizaciones de salud Enfermería asume la gestión del cuidado, en las que debe aplicar competencias de liderazgo y gestión, las cuales están directamente relacionadas con el trabajo en equipo, la toma de decisiones, y la planificación, entre otros elementos. Un estudio internacional realizado en el Hospital Universitario Regional del Norte de Paraná, Brasil, determinó que el estilo percibido por los profesionales de Enfermería, en su mayoría es "participativo" (33), lo que difiere de los hallazgos de este estudio, ya que en el servicio de Urgencia la mayoría de dichos profesionales percibe el estilo " $\mathrm{Di}$ rector". Esto puede tener relación con el tipo de atención que se da en este Servicio Clínico, el cual presenta un nivel de complejidad más elevado, producto del riesgo vital que tienen algunos usuarios, así como también una demanda mayor, requiriendo precisión y rapidez en las acciones, lo que provoca un ambiente en que los líderes deben definir los papeles y las tareas de los poderdantes y también supervisarlos de cerca. A diferencia de Urgencia, en Cirugía y Medicina se percibe predominantemente el estilo "Entrenador". En éstos la estadía de los usuarios es prolongada, lo que a su vez provoca una atención más tranquila, incitando al líder a una supervisión mucho más escasa, definiendo papeles y tareas, pero también buscando sugerencias e ideas de sus seguidores. En estos servicios puede existir una inclinación hacia la rutina y desmotivación, siendo necesario el soporte y alabanza para la construcción de autoestima, y la participación en la toma de decisiones para la restauración del compromiso. Sin considerar la variable servicio clínico, De
Melo y Schlindwein (34) en su artículo de revisión de liderazgo proponen una tendencia hacia un liderazgo auténtico/participativo, con enfoque en la comunicación, como forma de alcanzar mejores resultados en salud y superar los desafíos no lineares con una única causa.

Gené y Contel (35) plantean que la incidencia de los Estilos de liderazgo sobre la Satisfacción Laboral de un equipo de trabajo representa un $70 \%$ del clima organizacional que perciben las personas. Uno de los factores que, según el mismo autor, influye de manera positiva en la Satisfacción Laboral corresponde a la responsabilidad, entendida como la percepción de los miembros de un equipo sobre la delegación de autoridad y en qué medida sienten que la responsabilidad del resultado depende de ellos. En contraste a lo observado en esta investigación, se observó con respecto a la satisfacción laboral, clasificada en satisfecho e insatisfecho, que la mayoría de los profesionales de Enfermería se encuentran satisfechos con el estilo de liderazgo entrenador e insatisfechos con el estilo delegador, ya que dichos profesionales, en su mayoría jóvenes y con poca experiencia, aún no creen alcanzar niveles de madurez que les permitan realizar sus tareas de forma eficiente y sin necesidad de supervisión directa, motivo por el cual relacionan satisfacción laboral con un estilo de liderazgo que les permita establecer una comunicación de tipo bidireccional con sus líderes brindando sugerencias e ideas, pero sin involucrarse directamente en la toma de decisiones.

Los profesionales de Enfermería que perciben poca y nada satisfacción laboral, se correlacionan con los estilos Delegador y Director respectivamente, mientras que la percepción del nivel "bastante satisfecho" se correlaciona con los estilos participativo y entrenador. Así mismo es posible afirmar que la percepción de la satisfacción laboral de los profesionales de Enfermería categorizada como satisfechos, se asocia significativamente con los estilos de liderazgo entre- 
nador y participativo, mientras que aquellos, categorizados como insatisfechos laboralmente perciben como estilos de liderazgo predominante aquellos referidos a director y delegador. Dichos resultados son avalados por diversas investigaciones realizadas por las universidades de Ohio y Michigan, las cuales mostraron que los líderes que adoptan estilos entrenador y participativo tienden a lograr mejor desempeño y satisfacción de sus subordinados, debido a que subrayan las relaciones personales, se interesan particularmente en las necesidades de sus seguidores y aceptan las individualidades del grupo(36). Según Yáñez et al. un elemento crucial para el liderazgo efectivo es que los líderes cuenten con la confianza de sus trabajadores (37).

$\mathrm{Al}$ asociar Satisfacción Laboral y Estilo de liderazgo percibido, según el tipo de usuario que atienden los profesionales de Enfermería de este estudio, se encontró una asociación significativa sólo en la atención de adultos $(\mathrm{p}<0,01)$. Con respecto a la atención de niños, no existe asociación positiva entre las variables en estudio.

Finalmente es importante señalar que, como lo propone el modelo de Hersey y Blanchard, no existe un Estilo de liderazgo óptimo. Por el contrario, el estilo dependería directamente del nivel de preparación, también denominado nivel de madurez de los seguidores en los que el líder pretende influir, a fin de lograr la consecución de objetivos (21) y como lo propone un estudio mexicano, la satisfacción laboral está muy relacionada con tipo de supervisión, comunicación, autonomía y liderazgo en las autoridades de Enfermería (23).

Las enfermeras(o) gestoras(es) pueden influir ejerciendo un estilo de liderazgo adecuado a la situación y de esta manera aumentar la motivación y satisfacción laboral de su personal; como indica el estudio español realizado por Aguilar et al., el estilo de liderazgo que se adopte puede influir en el perfil de valores que tomen los seguidores o subordinados (28); por tanto como concluyen en su estudio Rodríguez et al., resulta primordial prestar atención a los estilos de liderazgo de los diferentes gestores de unidades o de grupos, dado que es un elemento dinamizador importante en las organizaciones (38).

\section{REFERENCIAS}

1. Marriner-Tomey A. Administración y Liderazgo en Enfermería. 5a ed. Editorial: Harcourt Brace.1998. p. 273-349.

2. Marriner-Tomey A. Guía de Gestión y Dirección de Enfermería. 8a ed. Barcelona: Elsevier; 2009. 544 p.

3. Chiavenato I. Introducción a la teoría general de la administración. 7a ed. México D.F.: McGraw-Hill Interamericana; 2006. $298 \mathrm{p}$.

4. Parra S, Paravick T. Satisfacción Laboral en Enfermeras/os que trabajan en el sistema de atención médica de urgencia (SAMU). Cienc. enferm. 2002; VIII(2): 37-48.

5. Alargunsoro A.M. Satisfacción laboral en las enfermeras correturnos de $\mathrm{Mu}$ tualia durante el año 2011. Biblioteca Las Casas [Internet]. 2012 [citado 13 abril 2013]; 8(2): 1-30. Disponible en: http:// www.index-f.com/lascasas/documentos/ lc0656.pdf

6. Muchinsky PM. Psicología aplicada al trabajo. 6a ed. España: Thomsom; 2000. $271 \mathrm{p}$.

7. Galaz JF, Contreras P. La Satisfacción Laboral de los académicos mexicanos en una universidad estatal pública: la realidad institucional bajo la lente del profesorado. Benito de Juárez, México: ANUIES; 2002. 21-3.

8. Robbin S, Coulter M. Administración. Madrid, España: Prentice-Hall; 2006. p. 395-96.

9. Fernández B, Paravic T. Nivel de Satisfacción Laboral en Enfermeras de Hospitales Públicos y Privados de la Provincia de 
Concepción, Chile. Cienc. enferm. 2003; IX(2): 57-66.

10. Fiabane E, Giorgi I, Squazzin C, Argentero P. Work engagement and occupational stress in nurses and other healthcare workers: the role of organizational and personal factors. J Clin Nurs. 2013- Doi: 10.1111/jocn.12084.

11. Locke EA. De nature and causes of job satisfaction, En Dunnette MD, editor. Handbook of industrial and organizational psychology. Chicago: Rand-Mc Nally College; 1976. p. 1297-349.

12. Chiang M, Salazar M, Huerta P, Núñez. A. Clima organizacional y satisfacción laboral en organizaciones del sector estatal (Instituciones públicas). Desarrollo, adaptación y validación de instrumentos. Universum [Internet]. 2008 [citado $16 \mathrm{de}$ abril 2013]; 23(2): 66-85. Disponible en: http://www.scielo.cl

13. XVI Congreso Nacional de Enfermeras Gestión del cuidado: Expresión de una práctica profesional autónoma [disertación]. [La Serena]: Colegio de Enfermeras de Chile; 2007.

14. Milos P, Bórquez B, Larraín AI. La "Gestión del Cuidado" en la legislación chilena (II) Estado actual. Cienc. enferm. 2011; XII(3): 23-33.

15. Stogdill RM, Bass B. Handbook of leadership: A survey of the literatura. 3ra ed. New York: Simon-Schister; 1990.

16. Stodgill R. Personal Factors Associated with Leadership: A Survey of the Literature. Journal of Psychology. 1948; 25(1): 35-71.

17. Hellriegel D, Jackson S, Slocum J. Administración: un enfoque basado en competencias.10a ed. México, D.F.: Thomson; 2002. p. 427-31.

18. Schebella Souto de Moura G, Muller de Magalhaes AM, Dall'agnol C, Cavalcanti B, Dos Santos D. Liderazgo en enfermería: análisis del proceso de elección de jefes. Rev Lat Am Enfermagen. 2010; 18(6): 1-9.
19. Hersey P, Blanchard K, Johnson D. Management of Organizational Behavior: Utilizing Human Resources. 7a ed. New Jersey: Prentice-Hall; 1996. 627 p.

20. García I. La formación de clima psicológico y su relación con los estilos de liderazgo. Granada: Universidad de Granada, Departamento de psicología social y metodología de las ciencias del comportamiento; 2006. 112 p.

21. Hersey P, Blanchard K, Johnson D. Administración del Comportamiento Organizacional, Liderazgo Situacional. 7a ed. México: Prentice-Hall; 1998. 99 p.

22. Véliz L, Paravic T. Coaching educativo como estrategia para fortalecer el liderazgo en enfermería. Cienc enferm. 2012; XVIII(2): 111-17.

23. Alba A, Salcedo RA, Zárate R, Higuera F. Satisfacción laboral del personal de enfermería en el Hospital General de México. Rev Enferm Inst Mex Seguro Soc. 2008; 16(3): 155-60.

24. Negussie N, Demissie A. Relationship between leadership styles of nurse managers and nurses' job satisfaction in Jimma University specialized hospital. Ethiop J Health Sci. 2013; (1): 49-58.

25. Castro R. Midiendo la eficiencia de los hospitales públicos en Chile [Internet]. España: Instituto de Salud Pública Español; 2007 [citado 17 abril 2013]. 9p. Disponible en: http://www.expansiva.cl

26. Cantera J. NTP 2013: Satisfacción laboral: encuesta de evaluación [Internet]. España: Ministerio de Trabajo y Asuntos Sociales, Instituto Nacional de Higiene y Seguridad en el Trabajo [citado 16 abril 2013]. 5 p. Disponible en: http://www. insht.es

27. Briceño CE et al. Satisfacción laboral en el personal de enfermería del sector público. Revista Electrónica de Medicina Intensiva [Internet]. 2005; [citado 16 abril 2013]; 5(4): 1-6. Disponible en: http:// remi.uninet.edu/2005/05/REMIA030. pdf 
28. Aguilar-Luzón M, Calvo-Salguero A, García-Hita MA. Valores laborales y percepción del estilo de liderazgo en personal de enfermería. Salud pública México. 2007; 49(6): 401-07.

29. Ribera-Domene D, Cartagena de la Peña E, Reig-Ferrer A, Romá-Ferri MT, Sanz I, Caruana-Vañó A. Estrés laboral y salud en profesionales de enfermería. Estudio empírico en la provincia de Alicante. España: Secretariado de Publicaciones, Universidad de Alicante; 1993. 124 p.

30. Ríos-Risquez M, Godoy-Fernández C. Relación entre Satisfacción Laboral y Salud General percibida en Profesionales de Enfermería de Urgencias. Enferm Clín. 2008; 18(3): 134-41.

31. Aguirre D. Satisfacción laboral de los recursos humanos de enfermería: factores que la afectan. Rev haban cienc méd [Internet]. 2009 [citado 16 abril 2013]; 8(4). Disponible en: http://scielo.sld.cu

32. Díaz MS, Stimolo MI, Caro NP. Satisfacción laboral y Síndrome de desgaste Laboral en Enfermeros de Hospitales Públicos Córdoba-Argentina. Med Segur Trab [Internet]. 2010 [citado 16 abril 2013]; 56 (218): 22-38. Disponible en: http://scielo. isciii.es/pdf/mesetra/v56n218/original2. pdf

33. Da Silva M, Galvao C. Aplicación del Liderazgo Situacional en la Enfermería de Centro Quirúrgico. Rev Esc Enferm USP. 2007; 41(1): 104-12.

34. De Melo G, Schlindwein B. Liderazgo del enfermero: una revisión integradora de la literatura. Rev Lat Am Enfermagem. 2011; 19(3): 1-9.

35. Gene J, Contel J.C. Gestión en Atención Primaria: Incorporación de la Práctica Directiva en el Liderazgo Asistencial. Barcelona, España: Masson, S.A; 1999.

36. Robbins S. Fundamentos de Comportamiento Organizacional. 5a ed. Naucalpan de Juárez, México: Prentice-Hall; 1998. $254 \mathrm{p}$.

37. Yáñez R, Loyola G, Huenumilla F. La confiabilidad en el líder: un estudio sobre las enfermeras jefes de un hospital. Cienc. enferm. 2009; XV(3): 77-89.

38. Rodríguez-Gonzalo A, Muñoz-Lobo MJ, Marzo-Martínez A, Sánchez-Vicario F. Liderazgo en grupos de trabajo enfermeros. Percepciones. Enferm Clin. 2009; 19(4): 206-09. 